\title{
Conventional Computed Tomographic Calcium Scoring vs full chest CTCS for lung cancer screening: a cost-effectiveness analysis
}

Boxiang Jiang1', Philip A. Linden', Amit Gupta ${ }^{2}$, Craig Jarrett'', Stephanie G. Worrell', Vanessa P. Ho ${ }^{3}$, Yaron Perry ${ }^{1}$, Christopher W. Towe ${ }^{1,4^{*}}$ (D) and on behalf of University Hospitals' Research in Surgical Outcomes and Effectiveness (UH-RISES)

\begin{abstract}
Background: Conventional CTCS images the mid/lower chest for coronary artery disease (CAD). Because many CAD patients are also at risk for lung malignancy, CTCS often discovers incidental pulmonary nodules (IPN). CTCS excludes the upper chest, where malignancy is common. Full-chest CTCS (FCT) may be a cost-effective screening tool for IPN.

Methods: A decision tree was created to compare a FCT to CTCS in a hypothetical patient cohort with suspected CAD. (Figure) The design compares the effects of missed cancers on CTCS with the cost of working up nonmalignant nodules on FCT. The model was informed by results of the National Lung Screening Trial and literature review, including the rate of malignancy among patients receiving CTCS and the rate of malignancy in upper vs lower portions of the lung. The analysis outcomes are Quality-Adjusted Life Year (QALY) and incremental costeffectiveness ratio (ICER), which is generally considered beneficial when $<\$ 50,000 / Q A L Y$.

Results: Literature review suggests that rate of IPNs in the upper portion of the lung varied from 47 to $76 \%$. Our model assumed that IPNs occur in upper and lower portions of the lung with equal frequency. The model also assumes an equal malignancy potential in upper lung IPNs despite data that malignancy occurs 61-66\% in upper lung fields.

In the base case analysis, a FCT will lead to an increase of 0.03 QALYs comparing to conventional CTCS (14.54 vs 14.51 QALY, respectively), which translates into an QALY increase of 16 days. The associated incremental cost for FCT is $\$ 278$ ( $\$ 1027$ vs $\$ 748$, FCT vs CTCS respectively. The incremental cost-effectiveness ratio (ICER) is $\$ 10,289$ / QALY, suggesting significant benefit. Sensitivity analysis shows this benefit increases proportional to the rate of malignancy in upper lung fields.

(Continued on next page)
\end{abstract}

\footnotetext{
* Correspondence: christopher.towe@uhhospitals.org

${ }^{1}$ Department of Surgery, University Hospitals Cleveland Medical Center, Cleveland, $\mathrm{OH}$, USA

${ }^{4}$ University Hospitals Cleveland Medical Center, Division of Thoracic and Esophageal Surgery, 11100 Euclid Avenue, Cleveland, OH 44106-5011, USA

Full list of author information is available at the end of the article
}

(c) The Author(s). 2020 Open Access This article is licensed under a Creative Commons Attribution 4.0 International License, which permits use, sharing, adaptation, distribution and reproduction in any medium or format, as long as you give appropriate credit to the original author(s) and the source, provide a link to the Creative Commons licence, and indicate if changes were made. The images or other third party material in this article are included in the article's Creative Commons licence, unless indicated otherwise in a credit line to the material. If material is not included in the article's Creative Commons licence and your intended use is not permitted by statutory regulation or exceeds the permitted use, you will need to obtain permission directly from the copyright holder. To view a copy of this licence, visit http://creativecommons.org/licenses/by/4.0/ The Creative Commons Public Domain Dedication waiver (http://creativecommons.org/publicdomain/zero/1.0/) applies to the data made available in this article, unless otherwise stated in a credit line to the data. 
(Continued from previous page)

Conclusion: Conventional CTCS may be a missed opportunity to screen for upper lung field cancers in high risk patients. The ICER of FCT is better than screening for breast cancer screening (mammograms $\$ 80 \mathrm{k} / \mathrm{QALY}$ ) and colon cancer (colonoscopy $\$ 6 \mathrm{k} / \mathrm{QALY}$ ). Prospective studies are appropriate to define protocols for FCT.

Keywords: Lung cancer screening, Conventional computed tomographic calcium scoring, Full chest calcium scoring scan, Cost-effectiveness analysis, Coronary artery disease

\section{Background}

Conventional computed tomographic calcium scoring (CTCS) is a radiographic study frequently used to screen for coronary artery disease (CAD) in asymptomatic patients $[1,2]$. Non-cardiac findings are routinely identified on CTCS, including, most commonly, incidental pulmonary nodules (IPN), which are identified in 10-18\% of patient [3-5]. Prior studies have shown that work up of these IPNs leads to improved lung cancer mortality [3]. The benefit in lung cancer mortality is limited to the cancers in the lower lung fields. CTCS only images the mid and lower chest and does not include the upper lung fields. Observational studies suggest that pulmonary nodules are more common in the upper lung fields, as primary lung cancers $[6,7]$. With this in mind, we believed CTCS may be a missed opportunity to scan the entire lung field for the purposes of identifying more "incidental" lung cancers.

Lung cancer is the leading cause of cancer death in the United States [8]. Based on the success of the National Lung Cancer Screening Trial (NLST) [8], the United States Preventive Service Task Force (USPSTF) recommends screening for lung cancer with low dose computed tomography (LDCT) in patients age 55-80 years who are current smokers (with a 30 pack-year smoking history) or have quit within 15 years [9]. Although lung cancer screening was found to be beneficial in this cohort, it is unclear if there is benefit in other populations.

We hypothesized that adding upper lung field to a calcium scoring test to image the "full chest" (FCT) was more cost-effective than conventional CTCS to screen for CAD and lung cancer. We performed a decision analysis to analyze the potential cost/benefit of the FCT study relative to $\mathrm{CTCS}$.

\section{Methods}

A cost-effectiveness decision tree model (Fig. 1) was built using Treeage Pro 2018 (Williamstown, MA). We assumed a hypothetical population with an average age of 50 years who required a screening CTCS to screen for CAD. Patients would either receive a CTCS or FTS as their primary coronary imaging method. The rate of IPN was modeled based on literature review [3-5]. The management of IPNs found on imaging was modeled using data from NLST [8]. IPNs were managed with either invasive procedures for diagnosis or followed by serial imaging. The complications of the invasive procedures were categorized as death, major complications, minor complications, or event free survival. The utility of patients ranges from 0 (death) to 1 (perfect health). A disutility was applied to major and minor complications in the post-procedure periods. If cancer was diagnosed, appropriate cancer treatment was implemented and outcomes were based on NLST data [8]. The outcome of the model was effectiveness and cost. Effectiveness was defined as quality adjusted life year (QALY) and cost reported in dollars $(\$)$, and incremental cost-effective ratio (ICER) was calculated from these outcomes.

\section{Missed pulmonary nodules (MPN)}

In CTCS, some pulmonary nodules are not identified due to the limited lung volume included in this imaging modality. For the decision analysis, we assume that the amount of the MPN were proportional to the unscanned lung volume. Because CTCS only includes the lower half of the lung field, we assumed that the amount of MPN were equal to the identified IPNs in CTCS. This may underestimate the true number of IPNs as some studies suggest higher rate of IPNs in the upper lung fields [6, 7]. Despite data regarding the malignant potential of IPN in CTCS $[3,5]$, the malignancy rate of the MPN is unknown. We assumed that the MPN malignancy rate was the same as the identified IPN, despite some suggestions that lung cancer is more common in the upper lung fields. Patient who received a CTCS and had MPNs were categorized as having either benign disease or malignancy. Those with malignancy were assumed to be present with lung cancer at later stages and received lung cancer treatment.

\section{Variables}

The key variables of the model included the probabilities of incidental nodules in patients receiving CTCS and the rate of malignancy found in these nodules. These variables are shown in Table 1 . The rates of invasive procedures and complications from these procedures were modeled from NLST and are also listed.

\section{Base case analysis}

The base case analysis represents the best estimation of the "real" difference between patients receiving CTCS 


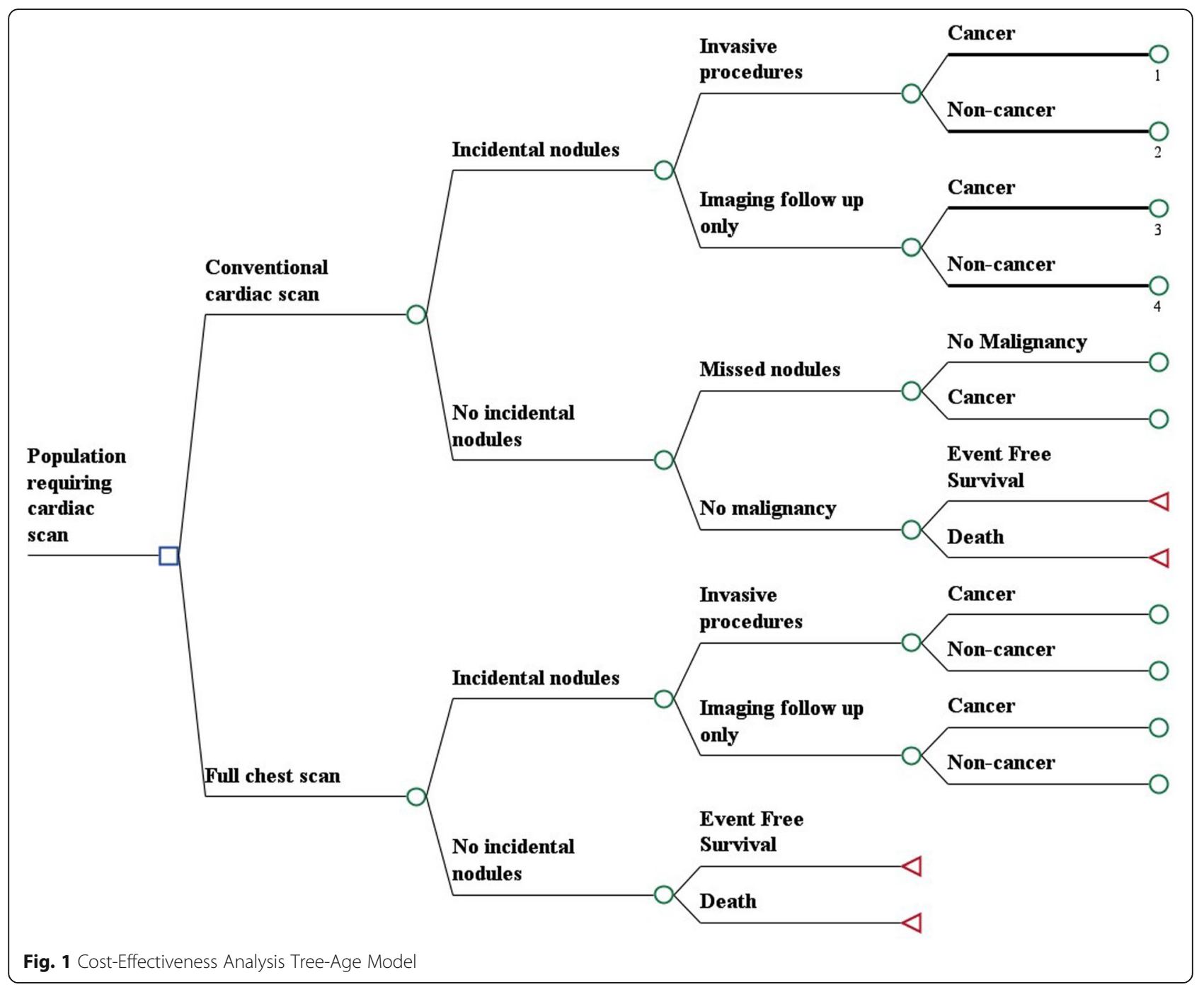

and FTS. Results of the base case analysis were effectiveness and cost. A protocol exhibited dominance if it was both life-saving and cost saving. If dominance was not achieved, ICER was calculated. We used ICER of $\$ 50$, 000/QALY as the societal threshold of cost-effectiveness.

\section{Monte Carlo simulation and sensitivity analysis}

A Monte Carlo simulation of 10,000 iterations was performed to test the variability of the model. The major variables were randomly re-sampled in a reasonable range as listed in Table 1. Results are reported as median and 1090th percentile. Single-variable sensitivity analyses were also performed for percentage of MPNs and malignancy rate of MPNs.

\section{Results}

Base case analysis

In the base case analysis, FCT is more cost-effective than CTCS, however, FCT does not dominate CTCS.
FCT saves an additional 0.03 QALY (14.54 vs 14.51 QALY, FCT vs CTCS respectively) with an additional cost of $\$ 278$ ( $\$ 1027$ vs $\$ 748$, FCT vs CTCS respectively). The ICER was calculated to be $\$ 10,289$ / QALY.

\section{Monte Carlo simulation}

A Monte Carlo simulation of 10,000 iterations was performed to assess the variability of the model. The median effectiveness is 14.54 QALY for FCT (14.38 to $14.70,10$ th and 90th percentile) compared to 14.51 QALY for CTCS (14.34 to 14.67, 10th and 90th percentile), confirming the benefit found in the base case analysis. The median cost for FCT and CTCS are $\$ 1023$ and $\$ 747$, respectively. The median ICER was $\$ 10,447$ /QALY (\$8039/QALY to \$13,186/QALY, 10th and 90th percentile). These results are summarized in Table 2. 
Table 1 Major variables used in the model to analyze the cost effectiveness of full chest CT vs conventional calcium score CT (CTCS)

\begin{tabular}{|c|c|c|c|}
\hline Possibilities & Base Case & Range & Reference \\
\hline Percentage of patients with IPNs on CTCS & $11.25 \%$ & N/A & [3] \\
\hline Percentage of patients with MPNs on CTCS & $11.25 \%$ & $11.25-14.40 \%$ & {$[3-5]$} \\
\hline Malignancy rate of IPNs & $3.67 \%$ & N/A & [3] \\
\hline Malignancy rate of MPNs & $3.67 \%$ & $3.67-5.5 \%$ & [3] \\
\hline Percentage of IPNs had invasive diagnostic procedure & $6.1 \%$ & N/A & [8] \\
\hline Percentage of IPNs followed with imaging only & $93.9 \%$ & N/A & [8] \\
\hline Any complications caused by invasive diagnostic procedure & $23.9 \%$ & N/A & [8] \\
\hline Major complications after invasive diagnostic procedure & $8.1 \%$ & N/A & [8] \\
\hline Mortality caused by invasive diagnostic procedure & $1.5 \%$ & $\mathrm{n} / \mathrm{a}$ & [8] \\
\hline Lifetime cancer recurrence rate & $33 \%$ & $\mathrm{n} / \mathrm{a}$ & {$[10]$} \\
\hline Utility & Base Case & Range & Reference \\
\hline Baseline utility & 0.845 & $0.838-0.854$ & {$[3,11-13]$} \\
\hline After lung cancer diagnosis & 0.62 & $0.31-0.83$ & {$[3,11-13]$} \\
\hline Major complication after invasive procedure & 0.5 & $0.4-0.7$ & {$[3,11,12]$} \\
\hline Minor complication after invasive procedure & 0.7 & $\mathrm{n} / \mathrm{a}$ & {$[3,11,12]$} \\
\hline Cost (in 2018 dollar value) & Base Case (\$) & Range (\$) & Reference \\
\hline CTCS & 382 & $156-467$ & [3] \\
\hline Invasive procedure & 12,321 & $638-18,970$ & {$[11,12,14]$} \\
\hline Imaging follow-up & 954 & $\mathrm{n} / \mathrm{a}$ & {$[11,12,14]$} \\
\hline Treating major complications & 6524 & $3262-19,678$ & {$[11,12,14]$} \\
\hline Treating minor complications & 622 & $311-933$ & {$[11,12,14]$} \\
\hline Cancer treatment & 12,217 & $6109-32,304$ & {$[11,12,14]$} \\
\hline
\end{tabular}

\section{Sensitivity analysis}

Sensitivity analyses of the malignancy rate of MPNs were performed. If the malignancy rate of MPNs is higher than $1.59 \%$, FCT becomes more cost-effective than CTCS (ICER < \$50,000/QALY). The ICER of FCT decreases with increased malignancy rate of MPNs (Fig. 2). However, the model is not sensitive to the rate of MPNs. The ICER of FCT continues to be at $\$ 10,289 /$ QALY when the rate of MPNs varies from 1 to $30 \%$.

\section{Discussion}

In our cost-effectiveness analysis, we showed that a full chest scan is cost-effective in patients requiring CT calcium scoring to screen for coronary artery disease because it saves lives from lung cancer. It saved 0.03 QALY (about 11 days of life) with an additional cost of $\$ 278$ for a calculated ICER of $\$ 10,289 /$ QALY. This result showed FCT was more cost-effective than CTCS. This

Table 2 Monte Carlo Simulation Result of 10,000 iterations

\begin{tabular}{lll}
\hline Median [10th - 90th percentile] & CTCS & FCT \\
\hline Effectiveness (QALY) & $14.51[14.34-14.67]$ & 14.54 [14.38-14.70] \\
Cost (\$) & $747[616-875]$ & 1023 [861-1190] \\
ICER (\$/QALY) & $10,447[8039-13,186]$ \\
\hline
\end{tabular}

benefit was further confirmed by Monte-Carlo simulation, which estimated the true ICER to be between $\$ 8039 /$ QALY and $\$ 13,186 /$ QALY. To put this result into perspective, implementation of mammogram for breast cancer screening has a reported ICER ranging from $\$ 60$, 000 to $\$ 80,000 /$ QALY [15]. Screening colonoscopy has an ICER of $\$ 15,000 /$ QALY which is comparable to the calculated ICER of FCT [16]. Both screening colonoscopy and mammogram are fully reimbursed by most insurances. While the NLST is currently the "gold standard" study for lung cancer screening, we believe that using the criteria within that study as the only criteria to screen for lung cancer is overly stringent. This study suggests that there is benefit to lung cancer screening in other populations as well. Coronary artery calcification is frequently identified in patients undergoing lung cancer screening with LDCT [17-19]. NLST data have shown that the presence of coronary artery calcification is associated with a 3 fold increase in cardiovascular death [18]. These studies suggest a concordance of CAD and lung cancer [17-19]. Therefore, adding patients who are receiving CTCS to a lung cancer screening cohort is reasonable on several levels.

The actual benefit of FCT is likely underestimated in this study. The actual malignancy rate of the MPNs in 


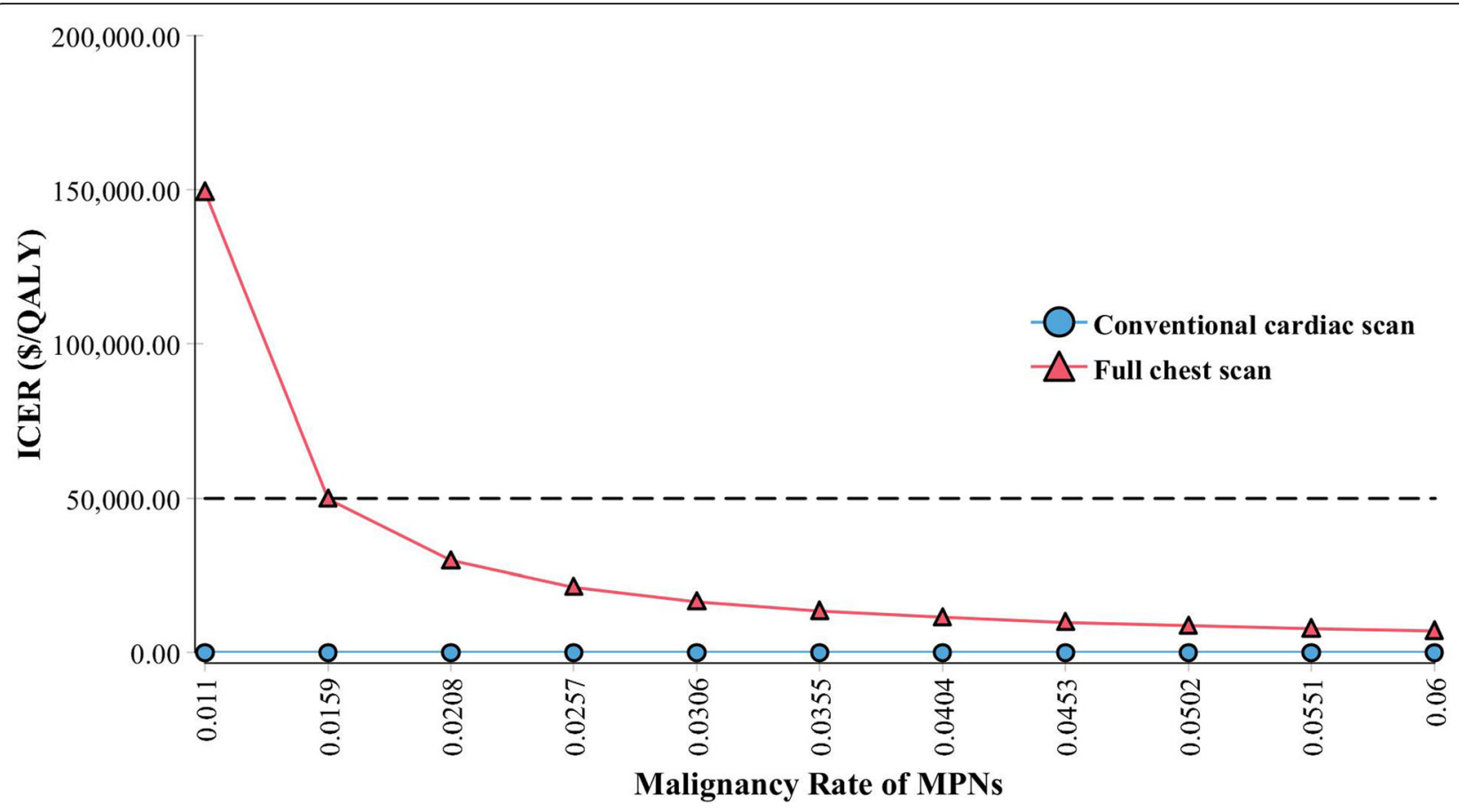

Fig. 2 Relationship of malignancy rate of missed pulmonary nodules vs Incremental Cost Effectiveness Ratio (ICER) of "full chest scan" vs conventional calcium score CT (FCT vs CTCS). Dashed line indicates the cost-effective threshold of $\$ 50,000 / \mathrm{QALY}$. When the malignancy rate > $1.59 \%$, FCT becomes cost-effective, ie ICER $<\$ 50,000 /$ QALY

this population was unknown, and estimation of this rate was intentionally conservative. Observational studies of pulmonary nodules based on locations suggest that the upper lung fields have more nodules than the lower lung fields and that upper lung nodules tended to have higher malignancy rate $[6,7]$. These observational studies were part of our rationale to modify existing CTCS scanning protocols. Interestingly, one study noted that one patient undergoing CTCS developed lung cancer without incidental finding on CTCS suggesting possible missed nodules [4]. In our model, we took a conservative approach in the base-case analysis by assuming an equal malignancy of upper and lower lung field nodules. Even with this assumption, the base-case analysis is cost-effective. Given the conservative nature of our estimates, the actual benefit of FCT may be even more cost-effective than our base-case analysis result. This was corroborated by the sensitivity analysis, which showed that the higher the malignant rate of the MPNs was, the lower the ICER of FCT would become.

Our study has several limitations. Foremost, the patient population who received calcium scoring imaging was different from the NLST inclusion criteria. NLST screened patients aged $55-75$ years old who were current smokers or a former smoker who quit less than 15 years prior and with at least 30 pack year smoking history [8]. The criteria for calcium score imaging is not specifically targeted to patient with history of tobacco use. Current expert consensus agreed that calcium score imaging is appropriate for asymptomatic patients who are $40-75$ year old with a 5-20\% 10 year atherosclerotic cardiovascular disease (ASCVD) risk [2]. The ASCVD risk is calculated based on age, sex, race, smoking status, blood pressure, cholesterol levels, smoking status and diabetic status [2]. Atherosclerotic cardiovascular disease shared risk factors with lung cancer which could explain the benefit of performing FCT on this population [17-19]. Current smoker and former smoker are reported to be around 28 and 39\% in patients requiring CTCS [3]. We concede that as tobacco rates decrease, the malignancy rate in IPN will also decrease, thereby erasing the benefit of FCT. While this is a significant limitation, the sensitivity analysis suggests that an FCT protocol for screening coronary artery disease with CT scan will have benefit until the malignancy rate in MPN falls below $1.5 \%$, which is much lower than current estimates [3]. Our model is not sensitive to the amount of the MPNs but only to the malignancy rate of the MPNs. In another word, the cost-effectiveness benefit of FCT derives from identifying malignant pulmonary nodules. As many studies have shown, the majorities of incidental pulmonary nodules found on CTCS are benign [4, 5]. Workup of benign findings will not translate into survival benefit which is proven in our model. 
As mentioned above, this study is an exploration of the potential benefit of FCT. In that regard, another weakness of this study is that FCT does not exist as an established imaging protocol. Imaging parameters are yet to be established. Our institution will be creating protocols for these studies and hope to standardize this for international adoption. We included a proposed FCT protocol in the supplemental material (Supplemental Figure 1). Other potential weaknesses of this study are that some outcomes of FCT are not modeled in the outcomes. For example, with increased lung field scanned, more incidental findings such as blebs and emphysematous change may found, and the burden of these findings are not included in the analysis. The increased workload of radiologists was not modelled in our analysis. Radiologists who read CTCS were required to recognize extracardiac findings as these were frequently found on the current CTCS [20]. FCS would identify more extracardiac findings which would require more work of the reading radiologist to characterize these findings. However, we expected the increased workload of FCT would be limited because these extracardiac findings of FCT were similar to that found on CTCS. From a practical standpoint, it would be very difficult to model the increased workload in our current model due to the lack of actual data. We felt it would be better explored in a prospective study. Lastly, the increased radiation exposure to the upper lung fields were not included. Recent studies have shown that the radiation exposure for CTCS is about $1 \mathrm{mSv}$ [21, 22]. Including additional apical lungs for FCT is likely resulting in a negligible increase of overall radiation exposure. Some studies have suggested high radiation exposure in cancer patient cohorts increase the risk of developing cardiovascular disease but the radiation exposure in the study was mainly from radiation therapy instead of diagnostic radiation [23].

The NLST included a strict inclusion criterion to reduce unnecessary screening and interventions. At the same time, it also prevented patients who might harbor lung cancers but did not meet the inclusion criteria from screening and early detection. Patients who were at high risk for lung cancers but did not meet the NLST criteria had a difficult time to obtain a screening LDCT and get insurance to pay for it [24]. This practically excluded them from being screened for lung cancer. Because lung cancer is by far the leading cause of cancer in the United States, we advocate for a new lung screening strategy to combat this disease. With the theoretical benefit for FCT in our study, we recommend implementation of this protocol. Once implemented, prospective study of the true effectiveness and cost could then be realized.

\section{Conclusion}

We concluded in our cost-effectiveness analysis that FCT was more cost-effective than CTCS for screening for lung cancer and coronary artery disease among patients with risk factors for coronary artery disease. This is the first study to show this potential benefit of FCT. Protocols and prospective studies are needed to further establish FCT and realize its full potential.

\section{Supplementary information}

Supplementary information accompanies this paper at https://doi.org/10. 1186/s12890-020-01221-8.

Additional file 1. Supplemental Figure 1. Proposed protocol for FCT.

\begin{abstract}
Abbreviations
CAD: Coronary artery disease; CTCS: Conventional computed tomographic calcium scoring; FCT: Full-chest CTCS; ICER: Incremental cost-effectiveness ratio.; IPN: Incidental pulmonary nodules; LDCT: Low dose computed tomography; MPN: Missed pulmonary nodules; NLST: National Lung Cancer Screening Trial; QALY: Quality-adjusted life year; USPSTF: United States Preventive Service Task Force
\end{abstract}

\section{Acknowledgements}

Not applicable.

\section{Authors' contributions}

BJ and CWT performed the literature review, designed the study, built the model, analyzed the results and wrote the manuscripts. PAL, AG, SGW, YP, CJ and VPH contributed to study design, interpretation of the results, and writing and review of the manuscript. All authors read and approved the final manuscript.

\section{Funding}

This study is funded by the Department of Surgery at University Hospitals and the University Hospitals' Research in Surgical Outcomes and Effectiveness $(\mathrm{UH}$ RISES). UH-RISES provides funding for purchasing Treeage software. These entities do not have any influence in the design of the study, analysis and interpretation of the data, or in writing the manuscript.

Availability of data and materials Not applicable.

Ethics approval and consent to participate

This study did not involve human subjects and was determined to be exempt from ethics and IRB approval by the University Hospitals Cleveland Medical Center Institutional Review Board.

Consent for publication

Not applicable.

\section{Competing interests}

Christopher Towe discloses that he is a consultant to Sig Medical, Zimmer Biomet, Medtronic and Atricure, but these relationships have not influenced the work or its conclusions.

The other authors have no competing interest.

\section{Author details}

${ }^{1}$ Department of Surgery, University Hospitals Cleveland Medical Center, Cleveland, OH, USA. ${ }^{2}$ Department of Radiology, University Hospitals Cleveland Medical Center, Cleveland, OH, USA. ${ }^{3}$ Deparment of Trauma and Acute Care Surgery, MetroHealth Hospital, Cleveland, OH, USA. ${ }^{4}$ University Hospitals Cleveland Medical Center, Division of Thoracic and Esophageal Surgery, 11100 Euclid Avenue, Cleveland, OH 44106-5011, USA. 
Received: 15 July 2019 Accepted: 22 June 2020

Published online: 06 July 2020

\section{References}

1. Greenland P, Bonow RO, Brundage BH, et al. ACCF/AHA 2007 clinical expert consensus document on coronary artery calcium scoring by computed tomography in global cardiovascular risk assessment and in evaluation of patients with chest pain: a report of the American College of Cardiology Foundation Clinical Expert Consensus Task Force (ACCF/AHA Writing Committee to Update the 2000 Expert Consensus Document on Electron Beam Computed Tomography) developed in collaboration with the Society of Atherosclerosis Imaging and Prevention and the Society of Cardiovascular Computed Tomography. J Am Coll Cardiol. 2007:49(3):378-402.

2. Hecht H, Blaha MJ, Berman DS, et al. Clinical indications for coronary artery calcium scoring in asymptomatic patients: expert consensus statement from the Society of Cardiovascular Computed Tomography. J Cardiovasc Comput Tomogr. 2017;11(2):157-68.

3. Goehler A, McMahon PM, Lumish HS, et al. Cost-effectiveness of follow-up of pulmonary nodules incidentally detected on cardiac computed tomographic angiography in patients with suspected coronary artery disease. Circulation. 2014;130(8):668-75.

4. Iribarren C, Hlatky MA, Chandra M, et al. Incidental pulmonary nodules on cardiac computed tomography: prognosis and use. Am J Med. 2008;121(11): 989-96.

5. Williams MC, Hunter A, Shah ASV, et al. Impact of noncardiac findings in patients undergoing CT coronary angiography: a substudy of the Scottish computed tomography of the heart (SCOT-HEART) trial. Eur Radiol. 2018; 28(6):2639-46.

6. Perandini S, Soardi G, Motton M, Oliboni E, Zantedeschi L, Montemezzi S. Distribution of solid solitary pulmonary nodules within the lungs on computed tomography: a review of 208 consecutive lesions of biopsyproven nature. Pol J Radiol. 2016;81:146-51.

7. Tammemagi MC, Schmidt H, Martel S, et al. Participant selection for lung cancer screening by risk modelling (the pan-Canadian early detection of Lung Cancer [PanCan] study): a single-arm, prospective study. Lancet Oncol. 2017;18(11):1523-31.

8. National Lung Screening Trial Research T, Aberle DR, Adams AM, et al. Reduced lung-cancer mortality with low-dose computed tomographic screening. N Engl J Med. 2011;365(5):395-409.

9. Moyer VA. Force USPST. Screening for lung cancer: U.S. preventive services task force recommendation statement. Ann Intern Med. 2014;160(5):330-8.

10. Uramoto H, Tanaka F. Recurrence after surgery in patients with NSCLC. Transl Lung Cancer Res. 2014;3(4):242-9.

11. Black WC, Gareen IF, Soneji SS, et al. Cost-effectiveness of CT screening in the National Lung Screening Trial. N Engl J Med. 2014;371(19):1793-802.

12. Deppen SA, Davis WT, Green EA, et al. Cost-effectiveness of initial diagnostic strategies for pulmonary nodules presenting to thoracic surgeons. Ann Thorac Surg. 2014;98(4):1214-22.

13. Trippoli S, Vaiani M, Lucioni C, Messori A. Quality of life and utility in patients with non-small cell lung cancer. Quality-of-life study Group of the Master 2 project in Pharmacoeconomics. Pharmacoeconomics. 2001;19(8): 855-63.

14. Dale CR, Madtes DK, Fan VS, Gorden JA, Veenstra DL. Navigational bronchoscopy with biopsy versus computed tomography-guided biopsy for the diagnosis of a solitary pulmonary nodule: a cost-consequences analysis. J Bronchology Interv Pulmonol. 2012;19(4):294-303.

15. Brown ML, Fintor L. Cost-effectiveness of breast cancer screening: preliminary results of a systematic review of the literature. Breast Cancer Res Treat. 1993;25(2):113-8.

16. Ran T, Cheng CY, Misselwitz B, Brenner H, Ubels J, Schlander M. Costeffectiveness of colorectal Cancer Screening strategies-a systematic review. Clin Gastroenterol Hepatol. 2019;17(10):1969-81 e1915.

17. Ravenel JG, Nance JW. Coronary artery calcification in lung cancer screening. Transl Lung Cancer Res. 2018;7(3):361-7.

18. Watts JR Jr, Sonavane SK, Snell-Bergeon J, Nath H. Visual scoring of coronary artery calcification in lung cancer screening computed tomography: association with all-cause and cardiovascular mortality risk. Coron Artery Dis. 2015:26(2):157-62.

19. Shemesh J, Henschke Cl, Shaham D, et al. Ordinal scoring of coronary artery calcifications on low-dose CT scans of the chest is predictive of death from cardiovascular disease. Radiology. 2010;257(2):541-8.
20. Johnson KM. Extracardiac findings on cardiac computed tomography: a radiologist's perspective. J Am Coll Cardiol. 2010;55(15):1566-8.

21. Patel AA, Fine J, Naghavi M, Budoff MJ. Radiation exposure and coronary artery calcium scans in the society for heart attack prevention and eradication cohort. Int J Cardiovasc Imaging. 2019;35(1):179-83.

22. Messenger B, Li D, Nasir K, Carr JJ, Blankstein R, Budoff MJ. Coronary calcium scans and radiation exposure in the multi-ethnic study of atherosclerosis. Int J Cardiovasc Imaging. 2016;32(3):525-9.

23. Yusuf SW, Venkatesulu BP, Mahadevan LS, Krishnan S. Radiation-induced cardiovascular disease: a clinical perspective. Front Cardiovasc Med. 2017;4:66.

24. Grannis FW Jr. National lung screening trial limitations and public health policy. Oncology (Williston Park). 2014;28(11):956-63.

\section{Publisher's Note}

Springer Nature remains neutral with regard to jurisdictional claims in published maps and institutional affiliations.
Ready to submit your research? Choose BMC and benefit from:

- fast, convenient online submission

- thorough peer review by experienced researchers in your field

- rapid publication on acceptance

- support for research data, including large and complex data types

- gold Open Access which fosters wider collaboration and increased citations

- maximum visibility for your research: over $100 \mathrm{M}$ website views per year

At BMC, research is always in progress.

Learn more biomedcentral.com/submissions 